\title{
Funga relacionada a Ocotea porphyria (Lauraceae): Xylaria (Xylariaceae, Ascomycota)
}

\author{
Funga related to Ocotea porphyria (Lauraceae): Xylaria \\ (Xyalariaceae, Ascomycota)
}

Medina, Patricia del V. ${ }^{*} ;$ Adriana I. Hladki ${ }^{1}$

1 Laboratorio Criptogámico, Fundación Miguel Lillo. Miguel Lillo 251, (T4000JFE) San Miguel de Tucumán, Tucumán, Argentina.

* Autor corresponsal: pmedina@lillo.org.ar

\section{RESUMEN}

Se presentan registros sobre los hongos xilariales que habitan en corteza y madera de Ocotea porphyria, un árbol endémico de la selva montana de Argentina y Bolivia. Se registran por primera vez seis especies del género Xylaria, incluyéndose descripciones, ilustraciones y comentarios sobre las mismas. Se proveen dos claves dicotómicas, una sobre los géneros y la otra sobre las especies presentes en este hospedante.

Palabras clave - Laurel del cerro; Xylariales; Yungas.

\begin{abstract}
Xylarial fungi records are present on Ocotea porphyria bark and wood. This is an endemic tree to the montane jungle of Argentina and Bolivia. Six species of the genus Xylaria are recorded for the first time, including descriptions, illustrations and comments on them. The dichotomous keys of genera and species are provided.

Keywords - Laurel del cerro; Xylariales; Yungas.

Ref. bibliográfica: Medina, P. del V.; Hladki, A. I. 2020. "Funga relacionada a Ocotea porphyria (Lauraceae): Xylaria (Xylariaceae, Ascomycota)". Lilloa 57 (1): 36-53. Fundación Miguel Lillo, Tucumán, Argentina. D.O.I.: doi.org/10.30550/j.lil/2020.57.1/3

> Recibido: 14/11/19 - Aceptado: 13/05/20

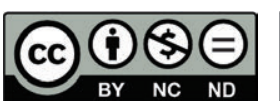

open 2 Access

> URL de la revista: http://lilloa.lillo.org.ar

- Esta obra está bajo una Licencia Creative Commons Atribución - No Comercial - Sin Obra Derivada 4.0 Internacional.
\end{abstract}




\section{INTRODUCCIÓN}

A comienzos del 2015, se iniciaron las investigaciones con el propósito de identificar, caracterizar y preservar la micobiota presente en Ocotea porphyria (Griseb.) van der Werff (Lauraceae), llamada vulgarmente "laurel del cerro". Esta es considerada una especie arbórea representativa de las Yungas Australes, y endémica de la región, cuyas características y distribución fueron detalladas en una contribución previa (Medina, Sir y Hladki, 2017).

Como resultado de numerosos viajes de campaña en áreas protegidas del Noroeste de Argentina (NOA), se realizó un relevamiento de la micobiota xilariacea presente sobre madera y corteza de este hospedante. Las primeras contribuciones de este grupo de investigación, se centraron particularmente en la identificación de las especies del género Hypoxylon Bull. (Medina et al., 2017). Esos novedosos registros se sumaron a los provenientes de relevamientos previos aportados por Hladki (2007), Hladki y Romero (2005, 2007, 2010), Sir, Hladki, Parrado, y Romero (2012), Medina, Sir, Grassi, y Hladki (2016) y Medina, Kuhnert, Hladki y Sir (“com. pers.", 2019).

En esta oportunidad las investigaciones se centraron en las especies de Xylaria Hill ex Schrank, siendo éste el género más diverso y complejo de la Familia Xylariaceae (Fournier, Flessa, Persoh, y Stadler, 2011; Stadler, Hawsworth, y Fournier, 2014). Sus miembros son un componente dominante de la comunidad endófita de los bosques alrededor del mundo y desempeñan una función importante como saprótrofos en estos ecosistemas (Rodrigues y Samuels, 1990; Rodrigues, Leuchtmann, y Petrini, 1993). En las Selvas del noroeste de Argentina existen más de 29 especies reportadas sobre distintos hospedantes nativos (Hladki y Romero 2010); sin embargo hasta el momento sólo se encuentran identificadas nueve especies creciendo sobre "laurel del cerro". Con la sospecha de que este número no reflejaba la realidad certeramente, se llevó a cabo un estudio profundo de las especies del género Xylaria, aportando en este trabajo, seis nuevos registros.

\section{MATERIALES Y MÉTODOS}

Las colecciones se realizaron en otoño (Mayo y Junio) de 2015, en los tres sectores latitudinales (zona norte, centro y sur) de las Yungas de Argentina, que se corresponden a condiciones diferentes de biodiversidad (Brown y Ramadori, 1989). En la zona norte de muestreo: Parque Nacional Calilegua, (Jujuy) y Reserva Provincial de Flora y Fauna Acambuco (Salta); en la zona centro: Parque Nacional El Rey (Salta) y en la zona sur: Parque Provincial "El Cochuna" (Tucumán) (Fig. 1).

Los especímenes examinados se encontraron sobre ramas caídas, y restos de madera y corteza extraídos con un cuchillo serrucho de grandes troncos caídos de $O$. porphyria. Las preparaciones, observaciones microscópicas, y la terminología empleada para las descripciones, se realizaron siguiendo a Hladki (2007).

Para la validación de la posición taxonómica de los hospedantes se consultó la base de datos del Missouri Botanical Garden (http://www.tropicos.org/); y para los hongos se consultó a http://www.indexfungorum.org/. 


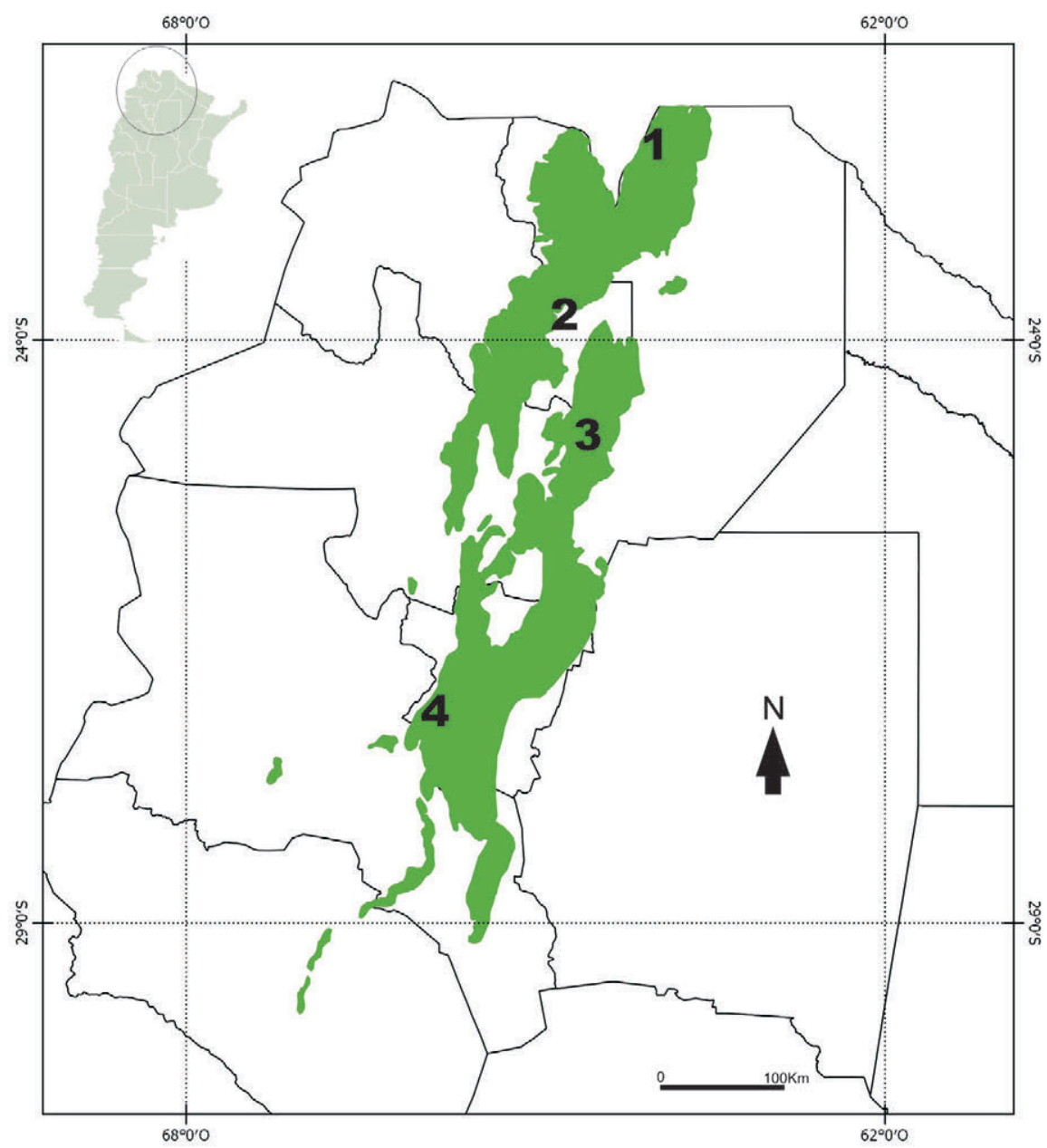

Fig. 1. Sitios de Muestreo: Zona Norte: 1) Reserva Provincial de Flora y Fauna Acambuco (Salta) y 2) Parque Nacional Calilegua (Jujuy). Zona Centro: 3) Parque Nacional El Rey (Salta). Zona Sur: 4) Parque Provincial "El Cochuna" (Tucumán).

Fig. 1. Sampling Sites: North Zone: 1) Acambuco Provincial Flora and Fauna Reserve (Salta) and 2) Calilegua National Park (Jujuy). Central Zone: 3) El Rey National Park (Salta). South Zone: 4) "El Cochuna" Provincial Park (Tucumán).

\section{RESULTADOS}

Se describen a continuación seis especies pertenecientes al género Xylaria sobre $O$. porphyria ampliando así a 21 el número de Xylariales (Familias Hyxpoxylaceae y Xylariaceae) que se conocen sobre este hospedante.

Xylaria culicicephala A.I. Romero \& Hladki, in Hladki \& Romero, Fungal Diversity 42: 80 (2010).

Fig. 2 A-G

Estromas gregarios, dispuestos en grupos pequeños sobre ramitas, filiformes diferenciados en una región fértil y un estípite, $2-5$ x 0,2-0,7 mm, castaño oscuro; estípite 
simple, no ramificado, subcilíndrico, erguido pero ocasionalmente curvado en el ápice, 0,5-1 mm long, castaño oscuro. Región fértil globosa a subglobosa, lisa, semejante a la cabeza de un mosquito por la presencia del ápice estéril corto, 0,5-1 mm long, cónico, central; superficie negruzca, lisa, brillosa; 1 a 3 peritecios desnudos por estroma, globosos, 0,7-1 mm diám; papilas ostiolares cónicas, rodeadas por un disco más alto que la superficie peritecial. Ascos 8-esporados, cilíndricos, 160-175 x 11-13 $\mu \mathrm{m}$, pie 85-98 $\mu \mathrm{m}$, aparato apical I+, urniforme, 5,5-7 x 4,5-6 $\mu \mathrm{m}$. Ascosporas elipsoidales e inequiláteras, naviculares con extremos estrechos redondeados, 28-34 x 10-12 $\mu \mathrm{m}$, castaño, surco germinativo recto, de igual longitud que la espora, en cara plana.

Fase asexual no observada (ver descripción en Hladki y Romero, 2010).

Material estudiado.- ARGENTINA. Prov. Jujuy. Dpto. Ledesma, P. N. Calilegua, $23^{\circ} 41^{\prime} 37,2$ 'S $64^{\circ} 52^{\prime 2} 21,2$ 'O; $1358 \mathrm{~m}$ snm, 26-V-2015, sobre ramita de O. porphyria; Medina y Hladki 218 (LIL 162010).

Materiales adicionales examinados.-ARGENTINA. Prov. Tucumán. Dpto. Tafí Viejo, Parque Biológico Sierra de San Javier, Cumbres de Taficillo, camino al puesto de Nina Velardez, El Balcón, 1090 m snm, 1-XII-1999, Hladki 2351 (LIL 150133). Dpto. Yerba Buena, Parque Biológico Sierra de San Javier, Horco Molle, 800 m snm, 6-IX-2006, Hladki 4005 (LIL 153008). Prov. Jujuy. Dpto. Ledesma, P. N. Calilegua, sobre Urera (L.) Gaudich, 26-V-2015; Sir 208 (LIL 152999).

Distribución geográfica.- Esta especie estaba citada únicamente en la provincia de Tucumán, Argentina por Hladki y Romero (2010), y en este trabajo se amplía su área de distribución a la provincia de Jujuy en el NOA.

Sustrato.- Sobre ramas en descomposición de eudicotiledóneas y en hojarasca (Hladki y Romero 2010). Se cita por primera vez sobre $O$. porphyria.

Observaciones.- Los especímenes de $X$. culicicephala encontrados sobre laurel del cerro presentan leves diferencias macro y microscópicas en comparación con el espécimen Tipo (4-8 mm). Los estromas examinados son de menor longitud (2-5 x $0,2-0,7$ vs $4-8 \times 0,2-0,6 \mathrm{~mm}$ ) con estípites de color castaño que ocasionalmente se curvan en el ápice, a diferencia de los estípites de color castaño oscuro, erguidos y rectos del Tipo. Además las colecciones jujeñas poseen peritecios levemente más pequeños (0,7-1 vs 0,9-1,2 mm diám). El aparato apical de los ascos es más pequeño, urniforme, I+ azul (5,5-7 x 4,5-6 vs 6,5-8 x 5-6,5 $\mu \mathrm{m})$ a diferencia del encontrado en el material Tipo que varía de urniforme a rectangular, I+ azul. Sin embargo las ascosporas del material aquí estudiado son levemente mayores que las del espécimen Tipo (28-34 x 10-12 vs $26-30 \times 10,5-12 \mu \mathrm{m}$ Tipo). 

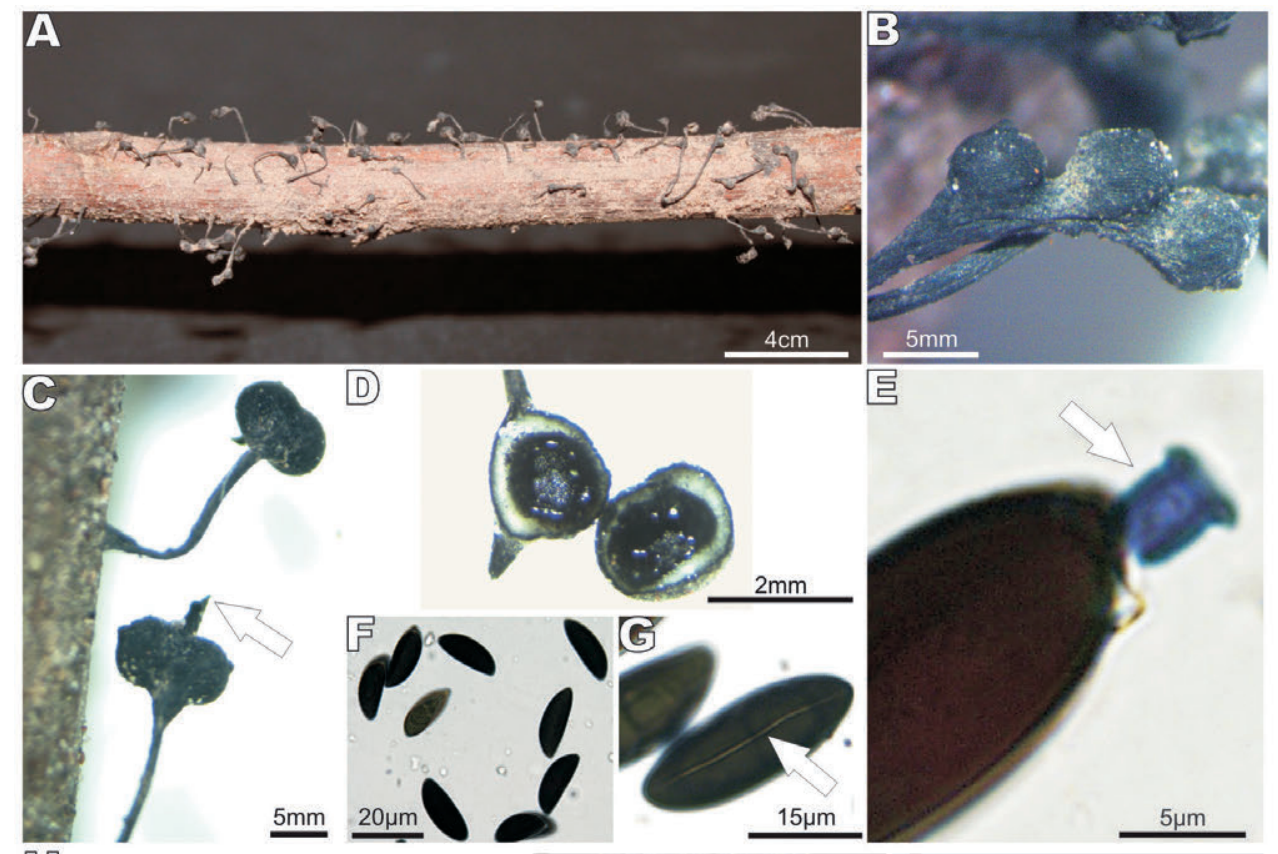

5

는
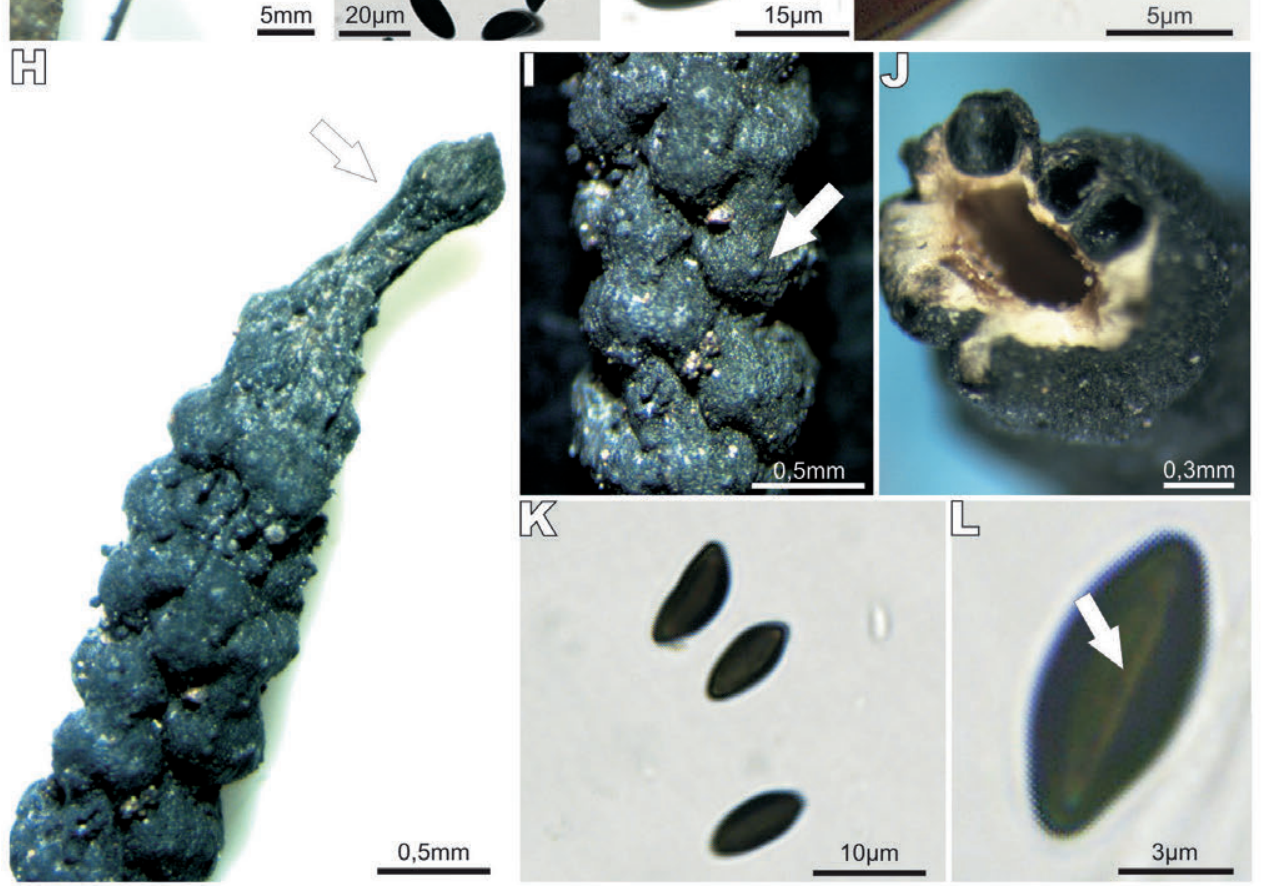

Fig. 2. Xylaria culicicephala. A) Aspecto general de los estromas sobre el sustrato. B) Vista de los peritecios en la parte superior del estroma. C) Vista en detalle del ápice estromático estéril, cónico y corto (flecha). D) Corte del estroma a la altura de la región fértil mostrando un peritecio globoso en sección longitudinal. E) Ápice ascal con aparato apical I+ (flecha). F) Ascosporas. G) Ascosporas, detalle del surco germinativo (flecha). Medina \& Hladki 218 (LIL 162010). Xylaria gracillima. H) Estroma, vista en detalle del ápice estéril (flecha). I) Contornos periteciales muy evidentes y ostíolos papilados (flecha). J) Corte del estroma a la altura de la región fértil, mostrando el interior hueco y los peritecios globosos en sección transversal sobre la periferia. K) Ascosporas. L) Ascosporas, detalle del surco germinativo recto (flecha). Medina \& Hladki 295 (LIL 162009).

Fig. 2. Xylaria culicicephala. A) Stromata on the substrate. B) Perithecia in the apex stroma. C) Detail of the sterile strongly conic apex. D) Longitudinal section of the stroma. E) Apical apparatus I+. F) Ascospores. G) Ascospores, detail of the germ slit. Medina \& Hladki 218 (LIL 162010). Xylaria gracillima. H) Stroma with sterile apex. I) perithecial contours conspicuous with prominent conical papillae ostioles. J) Transversal section of the stroma showing the hollow interior and globose perithecia. K) Ascospores. L) Ascospores straight germ slit (arrow). Medina \& Hladki 295 (LIL 162009). 
Xylaria gracillima (Fr.) Fr., Nova Acta R. Soc. Scient. upsal., Ser. 3 1(1): 128 (1851) [1855].

Fig. $2 \mathrm{H}-\mathrm{L}$

Estromas gregarios, dispuestos sobre madera, cilíndricos, diferenciados en una región fértil y estípite, $3 \times 1,5 \mathrm{~mm}$, castaño oscuro a negruzco; estípite corto, cilíndrico a subcilíndrico, 0,8-1 mm long. Región fértil cilíndrica, ápice agudo, estéril, con restos de tejido de ectostroma, contornos periteciales muy evidentes, 2-2,5 x 1-1,5 $\mathrm{mm}$. Peritecios globosos, 0,3-0,5 mm diám, papilas ostiolares conspicuas, cónicas. Ascos no observados. Ascosporas fusiformes a elipsoidales, inequiláteras, con extremos redondeados estrechos, 9-11 x 4-5 $\mu \mathrm{m}$, castaño oscuro, surco germinativo recto, ligeramente menor que la longitud de la espora.

Fase asexual no observada (ver descripción en Rogers, Callan, Rossman, Samuels, 1988; Rodrigues et al., 1993).

Material estudiado.- ARGENTINA. Prov. Salta. Dpto. Anta, P.N. El Rey 2443'33,7”S 64³9'50,5”O, $967 \mathrm{~m}$ snm, 28-V-2015, sobre rama caída de O. porphyria, Medina y Hladki 295 (LIL 162009).

Materiales adicionales examinados.—ARGENTINA. Prov. Tucumán. Dpto. Tafí Viejo, Parque Biológico Sierra de San Javier, Cumbres de Taficillo, camino al puesto de Nina Velardez, El Balcón, 1090 m snm, 20-VIII-99; Hladki 2669 (LIL 150801). Dpto. Yerba Buena, Parque Biológico Sierra de San Javier, Horco Molle, $800 \mathrm{~m} \mathrm{snm}$, 17-XI-99. Hladki 2777 (LIL 150767) y 17-VIII-99, Hladki 2602 (LIL 150829).

Distribución geográfica.- Esta especie fue inicialmente registrada en África, Brasil, Guyana y Venezuela (Dennis, 1961, 1970), luego en México (San Martín y Rogers, 1989, 1995), y en Argentina en las provincias de Catamarca y Tucumán (Agüero, Hladki, y Dios, 2010; Hladki y Romero, 2007). Se cita por primera vez para la provincia de Salta.

Sustrato.- San Martín (1992) la cita en suelo con abundante resto de material orgánico y Hladki y Romero (2007) la registran en ramitas caídas de eudicotiledóneas sin identificar. En este trabajo se cita por primera vez sobre $O$. porphyria.

Observaciones.- Existen algunas diferencias macro y microscópicas entre los estromas coleccionados sobre $O$. porphyria y los especímenes tucumanos descriptos por Hladki y Romero (2007); los estromas presentes en laurel son de menor tamaño (3 x $1,5$ vs $13-40$ x 1-1,5 mm) y sus ascosporas más cortas (9-11 x $4-5$ vs $9-13$ x $4-5 \mu \mathrm{m})$. 


\section{Xylaria grammica (Mont.) Mont., Nova Acta R. Soc. Scient. upsal.,} Ser. 31 (1): 128 (1851) [1855].

Fig. 3 A-E

Estromas solitarios sobre madera, claviforme, diferenciados en región fértil y estípite, 57-107 mm long, castaño oscuro. Estípite simple, no ramificado, subcilíndrico, 24-57 x 3,5-6 mm, castaño negruzco. Región fértil claviforme, constreñida en 2 o 3 regiones, ápice redondeado, superficie estromática lisa con fragmentaciones longitudinales subparalelas que se anastomosan espaciadamente delimitando fajas de ectostroma castaño claro, alternando con fajas de endostroma castaño, tornándose color grisáceo y negruzco respectivamente, a la madurez, $38-50$ x $9-13 \mathrm{~mm}$. Peritecios globosos, dispuestos en hileras, 0,6-0,9 mm diám, papila ostiolar diminuta, negra. Ascos 8esporados, cilíndricos, 68-184 x $5 \mu \mathrm{m}$, región fértil 33-89 $\mu \mathrm{m}$, pie 35-95 $\mu \mathrm{m}$, aparato apical I+, en forma de sombrero invertido. Ascosporas elipsoidales e inequiláteras, $12-13,5 \times 4,2-5 \mu \mathrm{m}$, castaño oscuro, surco germinativo recto de igual longitud que la espora, en la cara plana.

Fase asexual no observada (ver descripción en Hladki y Romero, 2005).

Materiales estudiados.-ARGENTINA. Prov. Salta. Dpto. Gral. José de San Martín, R. P. F. F. Acambuco, 2205’55,6”S 6356’31,0”O, 948 m snm, 22-V-2015, Medina y Hladki 51 (LIL 162011). Prov. Tucumán. Dpto. Chicligasta. P. P. El Cochuna, ruta 31 27 19'25,6”S 6555'41,1”O, 994 m snm, 18-VI-2015; Medina y Hladki 607 (LIL 162012).

Materiales adicionales examinados.- ARGENTINA. Prov. Tucumán. Dpto. Monteros, R. P. La Florida, ruta prov. 325, 700 m snm, 25-VIII-1999, Hladki 2710 (LIL 150787). Dpto. Tafí Viejo, Parque Biológico Sierra de San Javier, Cumbres de Taficillo, camino al puesto de Nina Velardez, El Balcón 1090 m snm, 24-V-1999, Hladki 2517 (LIL 150867).

Distribución geográfica.- África, Angola, Argentina (Catamarca, Jujuy, Misiones, Salta y Tucumán), Bolivia, Camerún, Cuba, Guyana, Indonesia, Nigeria, Nueva Guinea, Paraguay, Tailandia, Uganda y Venezuela (Agüero et al., 2010; Dennis, 1956, 1958a, 1961; Carroll, 1963; Rogers, Samuels y Callan, 1987; Spegazzini, 1909; Van der Gucht, 1995; Hladki, 2007; Hladki y Romero, 2005).

Sustrato.- Spegazzini (1909) la cita sobre troncos en descomposición en la provincia de Jujuy. Posteriormente Hladki (2007) la cita sobre grandes troncos de eudicotiledóneas sin corteza y con musgo, también creciendo en suelo con abundantes restos de madera y/o grandes hormigueros; asimismo la reporta creciendo sobre Podocarpus parlatorei Pilg. (Podocarpaceae), en la provincia de Salta. En el presente trabajo se registra por primera vez sobre $O$. porphyria.

Observaciones.- Nuestros materiales presentan pequeñas diferencias macroscópicas con los examinados por Hladki y Romero, 2005, donde observaron estromas 
ocasionalmente gregarios, dispuestos en ramillete, con la región fértil cilíndrica y constreñida en 2 o 3 partes, además de estípites acintados, ramificados y con base panosa. En cuanto a las características microscópicas, nuestras colecciones tienen peritecios más pequeños (0,6-0,9 vs $0,8-1,5 \mathrm{~mm})$, al igual que la región fértil de los $\operatorname{ascos}(33-89$ vs $65-90 \mu \mathrm{m})$.

\section{Xylaria multiplex (Kunze) Fr., Nova Acta R Soc. Scient. upsal., Ser. 3 1(1): 127. (1851) [1855].}

Fig. 3 F-K

Estromas cespitosos, en racimo naciendo de una base común, cilíndricos, diferenciado en región fértil y estípite, 3-23 x 0,3-1 mm, castaño. Estípite aplanado y surcado longitudinalmente, tomentoso en el tercio inferior, 6-9 x 1-2,8 mm, castaño. Región fértil cilíndrica, ápice estéril, en forma de cinta, 3-14 x 0,3-1 mm, castaño oscuro. Peritecios globosos, completamente inmersos en el estroma; papilas ostiolares diminutas, puntiformes, 0,5-0,6 x 0,4-0,5 mm, negro. Ascos 8-esporados, cilíndricos, 124-175 x 5,6 $\mu \mathrm{m}$, región fértil 70-85 $\mu \mathrm{m}$, pie 55-84 $\mu \mathrm{m}$, aparato apical $\mathrm{I}+$, rectangular, 1,5 x 1,6 $\mu \mathrm{m}$. Ascosporas, elipsoidales e inequiláteras, naviculares con extremos redondeados anchos, 10-11 x 4-5 $\mu \mathrm{m}$, castaño, surco germinativo recto ligeramente menor que la espora.

Fase asexual no observada (ver descripción Rodrigues et al., 1993, Van der Gucht, 1996).

Material estudiado. - ARGENTINA. Prov. Salta. Dpto. Anta, P. N. El Rey, 2443'33,4"S 64³9'51,9’'O, 973 m snm, 28-V-2015, sobre madera de tronco caído de O. porphyria, Medina y Hladki 304 (LIL 162007).

Materiales adicionales examinados.- ARGENTINA. Prov. Tucumán. Dpto. Capital, Jardín de la Fundación Miguel Lillo, 7-V-02, Hladki 2846 (LIL 150741). Dpto. Monteros, R. P. La Florida, ruta prov. 325, 700 m snm, 25-VIII-1999, Hladki 2706 (LIL 150790). Dpto. Tafí Viejo. Parque Biológico Sierra de San Javier, Cumbres de Taficillo, camino al puesto de Nina Velardez, El Balcón, junto a otras Xylaria, 1090 m snm, 1-III-1999, Hladki 2249 (LIL 151018).

Distribución geográfica.- En zonas tropicales y subtropicales. Argentina en las provincias de Salta y Tucumán (Hladki y Romero, 2007). Bolivia, Brasil, Colombia, Congo Francés, Ghana, Guayana Francesa, India, México, Papúa Nueva Guinea, Paraguay, Sierra Leona, Uganda, Venezuela (Miller, 1942; Dennis, 1956, 1958a, b, 1961, 1974; Thind y Waraitch, 1969; Pérez-Silva, 1975; San Martín y Rogers 1989).

Sustrato.- Sobre madera de eudicotiledóneas en estado de descomposición avanzada (Hladki y Romero, 2007). También fue citada sobre plantaciones de bambú, cafetales y en Craterispermum laurinum (Poir.) Benth (Dennis 1958a, 1974). En este trabajo se cita por primera vez sobre $O$. porphyria. 

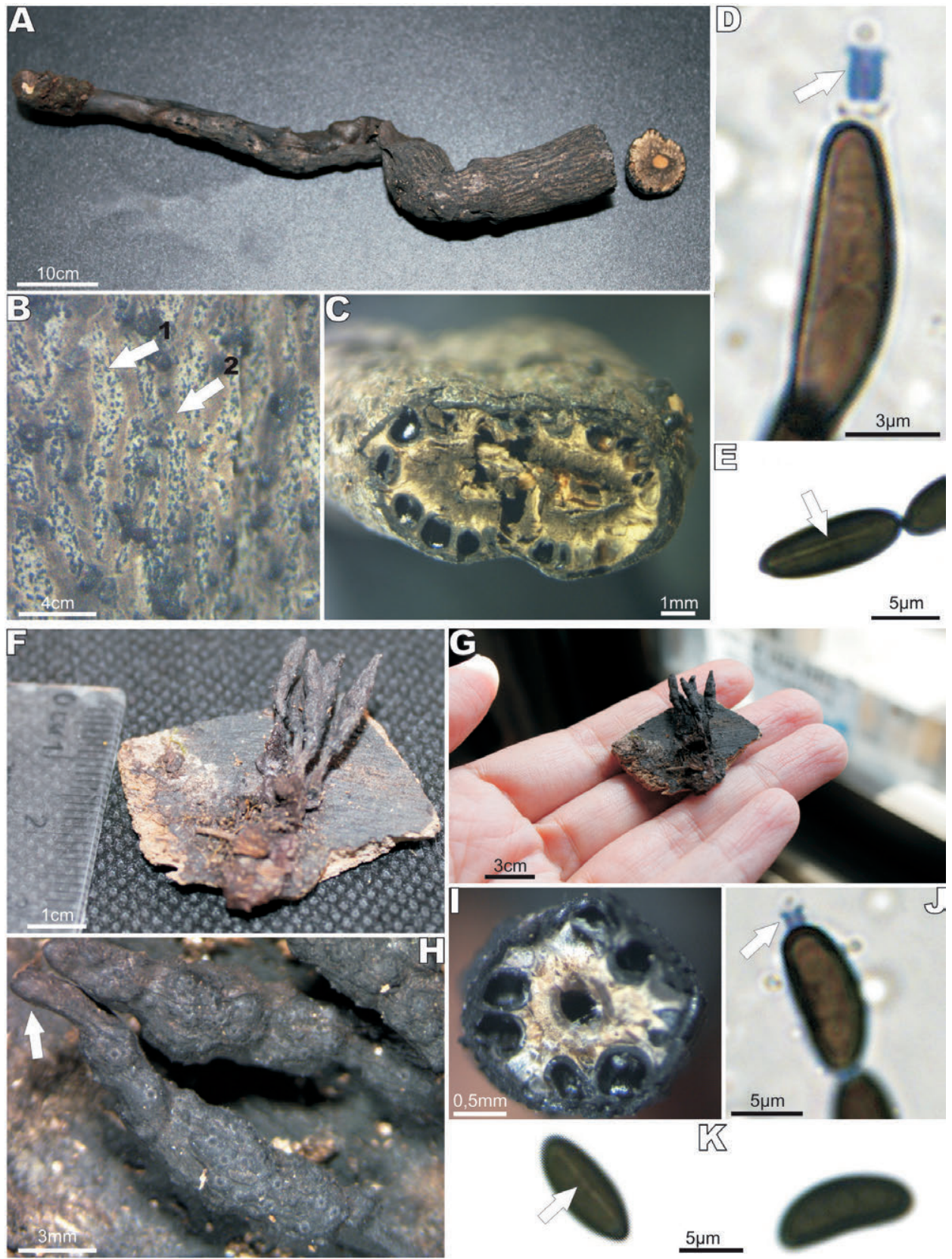

Fig. 3. Xylaria grammica. A) Aspecto general del estroma y vista del corte transversal del ápice. B) Superficie estromática, vista de las fajas longitudinales de ectostroma grisáceo (flecha 1) y de endostroma castaño (flecha 2), donde se observan las papilas ostiolares en hileras. C) Sección transversal del estroma, vista de los peritecios globosos. D) Ápice ascal con aparato apical I+ (flecha). E) Ascosporas, detalle del surco germinativo (flecha). Medina \& Hladki 51 (LIL 162011). Xylaria multiplex. F-G) Aspecto general de los estromas racimosos. H) Detalle de los ápices estromáticos estériles (flecha). I) Corte del estroma a la altura de la región fértil, mostrando el interior hueco y los peritecios globosos en sección transversal. J) Ápice ascal con aparato apical I+ (flecha). K) Ascosporas, detalle del surco germinativo (flecha). Medina \& Hladki 304 (LIL 162007).

Fig. 3. Xylaria grammica. A) Stroma and transversal section of the apex. B) Surface blackish with longitudinal grey (arrow 1) ectostromatal stripes and chestnut endostroma (arrow 2), with small papillate ostioles in rows. C) Transversal section with globose perithecia. D) Apical apparatus I+. E) Ascospores with germ slit. Medina \& Hladki 51 (LIL 162011). Xylaria multiplex. F-G) Stromata fasciculate. H) Apex sterile, acute. I) Transversal section of the hollow stroma, showing globose perithecia. J) Apical apparatus I+. K) Ascospores with germ slit. Medina \& Hladki 304 (LIL 162007). 
Observaciones. - Para la identificación del material se siguió la clave dicotómica de Hladki y Romero (2010), además, se contrastó con colecciones del NOA identificadas previamente como X. multiplex (Hladki y Romero, 2007). El especímen que crece sobre laurel del cerro presenta estromas en racimo a diferencia de otros especímenes con estromas cespitosos de esta especie que crecen sobre otros sustratos, sin embargo macroscópicamente, presentan todas las características diagnósticas que caracterizan a la especie. En tanto que la morfología y rangos de tamaño de peritecios, aparato apical y ascosporas, se ajustan a lo analizado en los materiales comparados.

\section{Xylaria cf. myosurus Mont., Annls Sci. Nat., Bot., sér. 4 3: 110 (1855).}

Fig. 4 A-E

Estromas solitarios o en grupos pequeños, errumpentes, cónicos a lanceolados, algunos más anchos en la base y agudizándose hacia un extremo, no ramificado, generalmente no diferenciado en una región fértil y estípite, o con estípite muy reducido (menor de $1 \mathrm{~mm}$ long), 7-9 x 1,8-2 mm, castaño negruzco. Región fértil con contornos periteciales poco evidentes, ápice agudo y papilas ostiolares prominentes mamiformes brillosas negras; superficie castaño negruzco. Peritecios globosos, inmersos en el estroma, 100-150 $\mu \mathrm{m}$ diám. Ascos 8-esporados, cilíndricos, 60-85 x 4-6 $\mu \mathrm{m}$, región fértil 58-76 $\mu \mathrm{m}$, pie 10-24 $\mu \mathrm{m}$, aparato apical $\mathrm{I}+$, en forma de cuña, 0,9 x 1,2 $\mu \mathrm{m}$, azul claro. Ascosporas elipsoidales e inequiláteras, extremos redondeados anchos, 6-7 x 3-4 $\mu \mathrm{m}$, castaño a castaño claro, surco germinativo recto ligeramente menor que la longitud de la espora.

Fase asexual no observada.

Material estudiado. - ARGENTINA. Prov. Jujuy. Dpto. Ledesma, P.N. Calilegua $23^{\circ} 41^{\prime} 57,6^{\prime \prime} \mathrm{S} 64^{\circ} 52^{\prime} 28,8^{\prime \prime} \mathrm{O}, 1206 \mathrm{~m}$ snm, 24-V-2015, sobre rama de O. porphyria, Medina y Hladki 158 (LIL 162013).

Materiales adicionales examinados.-ARGENTINA. Prov. Tucumán. Dpto. Chicligasta, P. P. El Cochuna, ruta prov. 331, Camping Samay, 1020 m snm, 14-VIII-1999, Hladki 2596 (LIL 150833). Dpto. Tafí Viejo, Parque Biológico Sierra de San Javier, Cumbres de Taficillo, camino al puesto de Nina Velardez, El Balcón 1090 m snm, 1-III-1999, Hladki 2254 (LIL 151015).

Distribución geográfica.- Brasil, Cuba, Guayana Francesa y Nueva Zelanda (Lloyd, 1920; Rick, 1935; Dennis, 1956; Martin, 1970; Rogers y Samuels, 1986); para la Argentina se la reportó en Catamarca y Tucumán (Agüero et al 2010; Hladki y Romero 2007). Mediante este trabajo se amplía el área de distribución en Argentina para la provincia de Jujuy.

Sustrato.- sobre ramas de eudicotiledóneas. Se cita por primera vez sobre O. porphyria. 

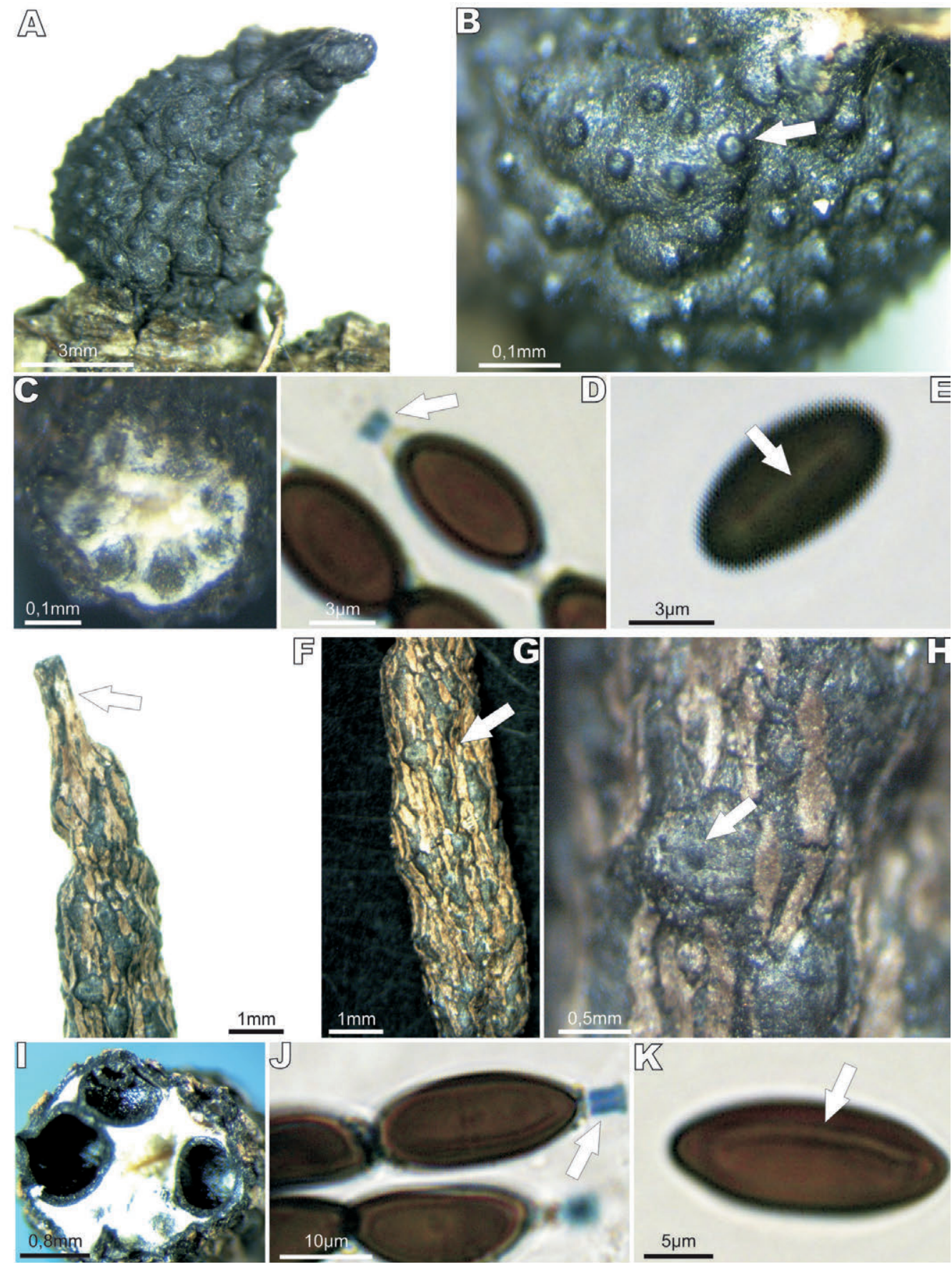

Fig. 4. Xylaria cf. myosurus. A) Aspecto general del estroma sobre el sustrato. B) Vista en detalle de las papilas ostiolares prominentes (flecha). C) Corte del estroma mostrando los peritecios globosos en sección transversal. D) Ápice ascal con aparato apical I+ (flecha). E) Ascospora, detalle del surco germinativo (flecha). Medina \& Hladki 158 (LIL 162013). Xylaria xylarioides. F) Detalle del ápice estromático estéril (flecha). G) Ectostroma en fajas longitudinales castaño claro (flecha). H) Ostíolos papilados (flecha). I) Corte del estroma a la altura de la región fértil, mostrando los peritecios globosos a ovados en sección transversal. J) Ápice ascal con aparato apical I+. K) Ascospora, detalle del surco germinativo. Medina \& Hladki 250 (LIL 162008).

Fig. 4. Xylaria cf. myosurus. A) Stroma on the substrate. B) Prominent conical papillae ostioles. C) Transversal section with globose perithecia. D) Apical apparatus I+. E) Ascospores with germ slit. Medina \& Hladki 158 (LIL 162013). Xylaria xylarioides. F) Stroma with sterile apex. G) Light brown ectostromatal stripes. H) Papillae ostioles. I) Transversal section showing globose to ovate perithecia. J) Apical apparatus I+. K) Ascospores with germ slit. Medina \& Hladki 250 (LIL 162008). 
Observaciones. - Nuestra colección presenta caracteres que lo asemejan a Xylaria myosurus. No obstante, al examinar materiales de la especie, que se coleccionaron en la provincia (ver Materiales adicionales examinados), se observó que los estromas y peritecios son considerablemente más pequeños (7-9 x 1,8-2 vs 12-20 x 1,5-2,5 mm; $100-150$ vs $220-275 \mu \mathrm{m}$ diám; respectivamente). Además las ascosporas también son de menor tamaño $(6-7 \times 3-4$ vs $8-9 \times 3,5-5 \mu \mathrm{m})$ y presentan surco germinativo que no cubre toda la longitud de la espora.

En contraste, “cf. $X$. myosurus". los caracteres descriptos para materiales neozelandeses, considerados como "cf. X. myosurus" (Rogers y Samuels, 1986), se ajustan en mayor medida a los especímenes que crecen sobre laurel del cerro.

Por lo tanto, se considera citar a Xylaria cf. myosurus como otra especie relacionada a este hospedante; hasta tanto se obtenga nuevas colecciones, cultivos y caracteres adicionales que confirmen la identidad de los especímenes.

\section{Xylaria xylarioides (Speg.) Hladki \& A.I. Romero, Fungal Diversity 42: 86 (2010).}

Fig. 4 F-K

Estromas solitarios, cilíndricos, diferenciados en región fértil y estípite, 8-15 x 3-5 $\mathrm{mm}$, castaño oscuro con fajas longitudinales de ectostroma castaño claro. Estípite subcilíndrico, no ramificado, tomentoso con pelos hifales, 1,7-3 x 0,2-2 mm, castaño oscuro. Región fértil con peritecios inmersos en el estroma, contornos periteciales poco evidentes, ápice estéril apiculado, castaño claro. Peritecios globosos a ovados, 0,5-0,8 mm, ostíolos finamente papilados, castaño oscuros. Ascos 8-esporados, cilíndricos, $220-300 \times 5,2 \mu \mathrm{m}$, región fértil 130-168 $\mu \mathrm{m}$, pie $90-132 \mu \mathrm{m}$, aparato apical I+, urniforme, 2,5-3 x 2,5-4 $\mu \mathrm{m}$. Ascosporas elipsoidales inequiláteras, naviculares, extremos redondeados, 17-20 x 6-8 $\mu \mathrm{m}$, castaño, surco germinativo recto, ligeramente menor que la longitud de la espora.

Fase asexual no observada (ver descripción en Hladki y Romero, 2010).

Material estudiado.- ARGENTINA. Prov. Salta. Dpto. Anta, P.N. El Rey 2443'23”S 64³9’41,9’O, 950 m snm, 28-V-2015, Medina y Hladki 250 (LIL 162008).

Materiales adicionales examinados.-ARGENTINA. Prov. Tucumán. Dpto. Chicligasta, Río Cochuna, 12-V-06, Hladki 2971 (LIL 150673). Dpto. Monteros, R. P. La Florida, ruta provincial 325, 24-XI-99, Hladki 2401 (LIL 150924). Dpto. Tafí del Valle, Tafí del Valle, ruta provincial 307, 19-IV-06, Hladki 2928 (LIL 153106).

Distribución geográfica.- Argentina, Buenos Aires como Hypoxylon xylarioides (Spegazzini, 1880), Tucumán como $X$. smilacicola y X. venosula (Spegazzini, 1909), Salta y Tucumán como X. xylarioides (Hladki y Romero, 2010); Brasil, São Paulo como $X$. venosula (Spegazzini, 1889); Nueva Zelanda como $X$. schereuderiana (Rogers y Samuels, 1986); Sudáfrica como X. schereuderiana (Miller, 1942) y X. papillata (Dennis, 1961). 
Sustrato.- Generalmente creciendo en ramitas de eudicotiledóneas Ripogonum scandens (J. R. Forst y G. Forst) y Solanum L. (Rogers y Samuels, 1986); Fussiaea sp y Smilax sp (Hladki y Romero, 2010; Spegazzini, 1880, 1881, 1909) y en tallos de monocotiledóneas sin identificar (Hladki y Romero, 2010).

En este trabajo se cita por primera vez sobre $O$. porphyria.

Observaciones.-Xylaria xylarioides es una especie común en las selvas del NOA.

Hladki y Romero (2010) en su estudio de numerosas colecciones de la región observaron una gran variación morfológica en los estromas de $X$. xylarioides, desde estromas sésiles subglobosos, estromas cónicos con pocos peritecios estipitados, hasta estromas subcilíndricos con numerosos peritecios; considerándola una variación intraespecífica. En el caso del material encontrado sobre laurel del cerro solamente se observaron estromas cilíndricos diferenciados en región fértil y estípite.

\section{Clave dicotómica de los géneros del Orden Xylariales presentes en $O$. porphyria}

1 Estromas sin pigmentos extraíbles (Fam. Xylariaceae) …................................. 2

1' Estromas con pigmentos extraíbles (Fam. Hypoxylaceae) ................................. 3

2 Estromas carbonosos con tejido interno que se disgrega dejando una estructura hueca a la madurez. Sin presencia de especies polimórficas ...... Kretzschmaria

2' Estromas no carbonosos y sin tejido interno que se disgrega dejando una estructura hueca a la madurez. Presencia de especies polimórficas ........ Xylaria

3 Estromas conspicuos esféricos, esféricos deprimidos, placentiformes, peltados, turbinados, claviformes o cilíndricos; sésiles o subsésiles, con tejidos internos formando anillos concéntricos intercalares claros y oscuros Daldinia

3' Estromas efusos, con tejido entre y por debajo de los peritecios, sólido homogéneo, generalmente no carbonoso Hypoxylon

\section{Clave dicotómica de las especies de las Familias Xylariaceae e Hypoxylaceae sobre $O$. porphyria}

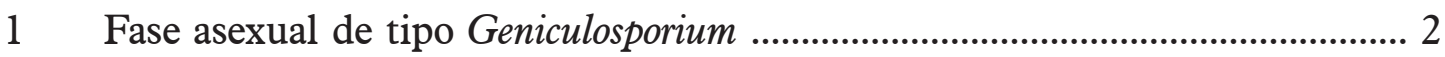

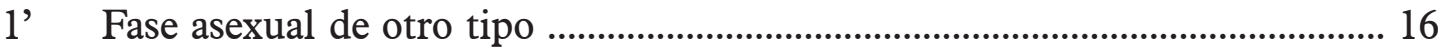

2 Estromas gregarios, fusionados, formando costras grandes compactas Kretzschmaria clavus

2' Estromas no fusionados en costras compactas .................................................... 3

3 Región fértil del estroma mayor de $5 \mathrm{~mm}$ de diám. ........................................ 4

3' Región fértil del estroma menor de $5 \mathrm{~mm}$ de diám. ........................................ 7

4 Superficie lisa y gruesa, textura dura y sin contornos periteciales evidentes, ocasionalmente con papilas ostiolares prominentes o restos de ectostroma 
4' Superficie rugosa y delgada, textura blanda, y/o con contornos periteciales evidentes 6

5 Estromas claviformes a fusiformes, hendidos longitudinalmente y enrollándose a la madurez; superficie amarillenta a castaña clara en fresco; ascosporas 21-28,5 $\mathrm{x} 6,5-8 \mu \mathrm{m}$, surco germinativo corto y oblicuo o sigmoide .............. X. telfairii

5. Estromas claviformes, constreñidos en 2 o 3 regiones; superficie negruzca con fajas longitudinales grisácea de ectostroma; ascosporas 12-13,5 x 4,2-5 $\mu \mathrm{m}$, surco germinativo largo y recto

$X$. grammica

6 Estromas cilíndricos claviformes; ascosporas con surco germinativo recto

X. curta

6' Estromas extremadamente variables en forma, generalmente fragariformes o moriformes; ascosporas con surco germinativo espiralado ................ X. anisopleura

7 Estromas compuestos de un estípite delicado y delgado donde se disponen pocos peritecios desnudos

7 $\quad$ Estromas con un estípite de mayor espesor con contornos periteciales parcial o totalmente inconspicuos

8 Estromas solitarios o en grupos pequeños, $4-8 \times$ x 0,2-0,6 mm, generalmente 1 peritecio por estroma, raramente 3 ; ascosporas naviculares con extremos estrechos redondeados, $28-34 \times 10-12 \mu \mathrm{m}$, castaño, surco germinativo recto

X. culicicephala

8' Estromas gregarios, $2-23 \times 1-3 \mathrm{~mm}$, numerosos peritecios por estroma; ascosporas naviculares con extremos redondeados, 30-36,5 x 10,5-13 $\mu \mathrm{m}$, surco germinativo ligeramente oblicuo X. melanura

9 Estromas con contornos periteciales conspicuos ............................................... 10

9' Estromas con contornos periteciales poco evidentes o nulos ........................... 11

10 Estromas generalmente paralelos al sustrato; región fértil y estípite en forma de cintas o subcilíndrico piloso; ascosporas naviculares con extremos redondeados anchos y apéndices hialinos cuando están inmaduras, castaño claro, 10,5-12 x $4-5 \mu \mathrm{m}$, surco germinativo recto y largo ........................ X. aff. ianthinovelutina

10' Estromas erguidos, región fértil y estípite cilíndrico; ascosporas fusiformes a elipsoidales, inequiláteras, con extremos redondeados estrechos, castaño oscuro, 9-11 x 4-5 $\mu \mathrm{m}$, surco germinativo recto, ligeramente menor que la longitud de la espora $X$. gracillima

11 Estromas variables en forma, fragariformes, lanceolados, moriformes, pero nunca cilíndricos 12

11' Estromas generalmente cilíndricos o raramente aplanados flabeliformes ...... 13

12 Estromas moriformes o fragariformes, con contornos periteciales evidentes; diferenciado en región fértil y estípite, ascosporas con surco germinativo espiralado $X$. anisopleura

12' Estromas cónicos a lanceolados, contornos periteciales poco evidentes, no diferenciado en región fértil y estípite; ascosporas con surco germinativo recto $X$. myosurus

13 Estromas fasciculados, superficie con fajas longitudinales de ectostroma negro, tornándose hueco a la madurez 14 
13' Estromas solitarios o en grupos pequeños, superficie castaño oscura con fajas longitudinales de ectostroma castaño claro

14 Estromas laxamente fasciculados, cilíndricos o aplanados flabeliformes, estípite cilíndrico liso; ascosporas naviculares, 14,5-17,5 x 4-7 $\mu \mathrm{m}$, surco germinativo recto a ligeramente oblicuo ocupando toda la longitud de la espora

$X$. fissilis

14' Estromas cespitosos, en racimo naciendo de una base común, cilíndricos; estípite aplanado y surcado longitudinalmente, tomentoso en el tercio inferior; ascosporas naviculares $10-11 \times 4-5 \mu \mathrm{m}$, surco germinativo recto ligeramente menor que la espora X. multiplex

15 Estromas solitarios, cilíndricos, diferenciados en región fértil y estípite corto menor de $3 \mathrm{~mm}$ long; ascosporas 17-20 x 6-8 $\mu \mathrm{m}$.......................... X. xylarioides

15' Estromas gregarios, cilíndricos con estípite bien desarrollado; ascosporas, 12$14,5 \times 5-6,5 \mu \mathrm{m}$ $X$. mellissi

16 Estromas hemisféricos pulvinados, con la base constreñida, turbinado a placentiforme, sésiles o estipitados Daldinia eschscholtzii

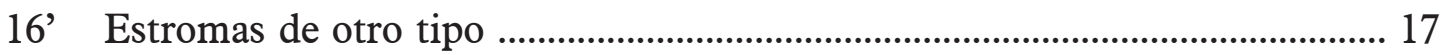

17 Ascosporas con surco germinativo levemente sigmoide a sigmoide ............ 18

17' Ascosporas con surco germinativo recto ....................................................... 19

18 Estromas glomerulares, efuso-pulvinado, con tendencia a peritecioide (acercándose a rosellinioide), ascosporas con surco germinativo levemente sigmoide a sigmoide de la longitud de la espora Hypoxylon lenormandii

18' Estromas efuso-pulvinados con montículos periteciales discretos a conspicuos, ascosporas con surco germinativo levemente sigmoide H. rickii

19 Ostíolos no rodeados por un disco elevado, levemente papilados H. cf. undulatum

19' Ostíolos umbilicados pero rodeados por un disco elevado 20

20 Estromas hemisféricos a deprimidos-esféricos, ostiolos umbilicados rodeados por un disco elevado fugaz H. ochraciotuberosum

20' Estromas glomerulados a efusos, con montículos periteciales conspicuos, ostiolos umbilicados pero rodeados por un disco elevado H. flavoargillaceum

\section{CONCLUSIÓN Y DISCUSIÓN}

Sir y colaboradores (2012) reportaron 49 taxones xilariaceos en áreas protegidas de la provincia de Tucumán; de los cuales dos crecían sobre estiércol y 22 sobre madera en estado avanzado de descomposición. Entre los hospedantes que pudieron ser identificados, observaron que Ocotea porphyria y Podocarpus parlatorei fueron los que presentaron una mayor diversidad. Posteriormente las investigaciones realizadas por Medina, Sir y Hladki (2017) y Medina, Kuhnert, Hladki y Sir (“Com. pers.”, 2019), sobre madera, corteza y ramitas de laurel del cerro lograron ampliar el número de especies del género Hypoxylon que habitan sobre este hospedante. 


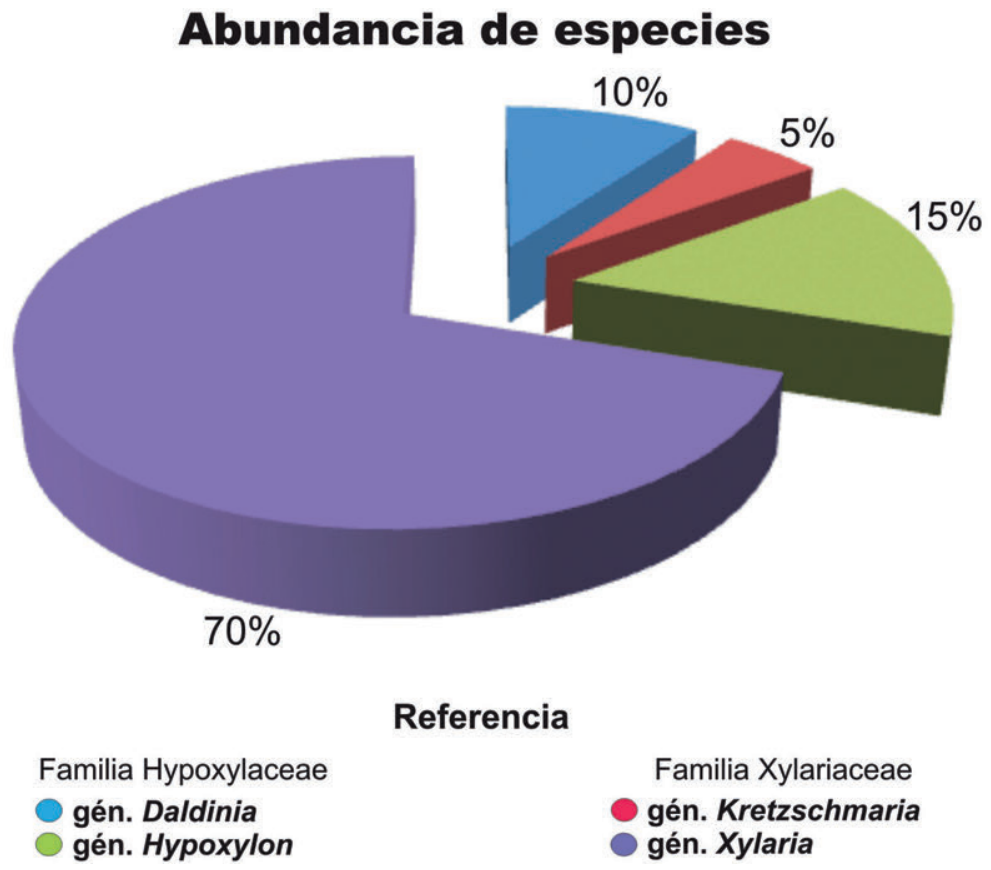

Fig. 5. Abundancia específica (expresada en porcentajes) de los géneros de Xylariales presentes sobre Ocotea porphyria.

Fig. 5. Specific abundance (expressed in percentages) of the Xylariales genus in Ocotea porphyria.

En este trabajo y como resultado del estudio de las especies del género Xylaria asociadas al laurel del cerro se registran por primera vez sobre este hospedante a: $X$. culicicephala, $X$. gracillima, $X$. grammica, $X$. multiplex, $X$. cf. myosurus y $X$. xylarioides. Además se amplía el área de distribución de $X$. culicicephala y $X$. cf. myosurus a la provincia de Jujuy y de $X$. gracillima a la provincia de Salta. Y finalmente, mediante estas seis nuevas contribuciones, se amplía el número de hongos xilariaceos que habitan sobre $O$. porphyria a 21 registros, observándose una predominancia de los géneros de la Familia Xylariaceae, que acumulan hasta el $75 \%$ de la riqueza total de xilariales descubiertas hasta el momento en relación a este hospedante (Fig. 5).

\section{AGRADECIMIENTOS}

Deseamos expresar nuestro agradecimiento a Parques Nacionales por su valiosa colaboración en los viajes de campaña; habiendo asistido con hospedaje, guía y conocimiento de las zonas examinadas. A la Fundación Miguel Lillo por haber facilitado los medios para la realización de este trabajo y al Dr. Esteban Sir (INBIOFIV-CONICET) por su colaboración en la realización del mapa de sitios de muestreo. 


\section{BIBLIOGRAFÍA}

Agüero, A. N., Hladki, A. I. y Dios, M. M. (2010). La Familia Xylariaceae (Xylariales) en la provincia de Catamarca (Argentina). Lilloa 47 (1-2): 3-9.

Brown, A. D. y Ramadori, E. D. (1989). Patrón de distribución, diversidad y características ecológicas de especies arbóreas de las selvas y bosques montanos del noroeste de la Argentina. Annales VI Congreso Forestal Argentino: 177-191.

Carrol, G. (1963). Studies in the flora of Thailand 24. Pyrenomycetes. Dansk Botanisk Arkiv 23: 101-114.

Dennis, R. W. G. (1956). Some Xylarias of Tropical America. Kew Bulletin 3: 401444.

Dennis, R. W. G. (1958a). Ascomycetes en Bolivia and North Argentina. Kew Bulletin 13: 153-154.

Dennis, R. W. G. (1958b). Some Xylosphaera of Tropical Africa. Revista de Biología 1 (3-4): 175-208.

Dennis, R. W. G. (1961). Xylarioideae and Thammomycetoideae of Congo. Bulletin du Fardin Botanique de I'État.a Bruxelles 31: 109-154.

Dennis, R. W. G. (1970). Fungus flora of Venezuela and adjacent countries. Kew Bulletin, add. ser. 3: 1-531.

Dennis, R. W. G. (1974). Xylariaceae from Papua and New Guinea. Bulletin Mensuel de la Société Linnéenne Lyon, numéro Spécial 43: 127-138.

Fournier, J., Flessa, F., Persoh, D. y Stadler, M. (2011). Three new Xylaria species from southwestern Europe. Mycological Progress 10: 33-52.

Hladki, A. I. (2007). La Familia Xylariaceae (Órden Xylariales), en la provincia de Tucumán. (Tesis Doctoral). Universidad Nacional de Tucumán, Argentina.

Hladki, A. I. y Romero, A. I. (2005). Contribución al estudio de las Xylariaceae de la República Argentina. III) Nuevos registros del género Xylaria. Lilloa 40 (12): 47-68.

Hladki, A. I. y Romero, A. I. (2007). Primeras citas del género Xylaria (Ascomycota, Xylariaceae) para la República Argentina. Darwiniana 45 (1): 28-44.

Hladki, A. I. y Romero, A. I. (2010). A preliminary account of Xylaria in the Tucumán Province, Argentina, with a key to the know species from the Northern Provinces. Fungal Diversity 42: 79-96.

Lloyd, C. G. (1920). Mycological Notes N 62. Mycological Writings 6: 904944.

Martín, P. (1970). Studies in the Xylariaceae: VIII. Xylaria and its allies. South African Fournal of Botany 36: 73-138.

Medina, P., Sir, E. B., Grassi, E. M. y Hladki, A. I. (2016). Nuevas citas del género Poronia (Xylariaceae, Ascomycota) para el Norte de Argentina. Lilloa 53 (1): 133-38.

Medina, P., Sir, E. B. y Hladki, A. I. (2017). Primeras citas del género Hypoxylon (Ascomycota, Xylariales) sobre Ocotea porphyria (Lauraceae). Lilloa 54 (2), 202209. DOI: https://doi.org/10.30550/j.lil/2017.54.2/10.

Miller, J. H. (1942). South African Xylariaceae. Bothalia 4: 251-272.

Pérez-Silva, E. (1975). El género Xylaria (Pyrenomycetes) en México, I. Boletín Sociedad Mexicana de Micología 9: 31-52. 
Rick, J. (1935). Monographia das Xylariaceas Riograndenses. Arquivos do Museu Nacional 36: 40-71.

Rodrigues, K. y Samuels, G. J. (1990). Preliminary study of endophytic fungi in a tropical palm. Mycological Research 94 (6): 827-830.

Rodrigues, K., Leuchtmann, A. y Petrini, O. (1993). Endophytic species of Xylaria: cultural and isozymic studies. Sydowia 45 (1): 116-138.

Rogers, J. D. y Samuels, G. J. (1986). Ascomycetes of New Zealand 8. Xylaria. New Zealand Fournald of Botany 24: 615-650.

Rogers, J. D., Samuels, G. J. y Callan, B. E. (1987). The Xylariaceae of the rain forest of North Sulawesi (Indonesia). Mycotaxon 29: 113-172.

Rogers, J. D., Callan, B. D., Rossman, A. Y. y Samuels, G. J. (1988). Xylaria (Sphaeriales, Xylariaceae) from Cerro de la Neblina Venezuela. Mycotaxon 31: 103-153.

San Martín, F. (1992). A mycofloristic and cultural study of the Xylariaceae of México. (Ph. D. Thesis), Washington State University, Pullman, Washington, USA.

San Martín, F. y Rogers, J. D. (1989). A preliminary account of Xylaria of México. Mycotaxon 34 (2): 283-373.

San Martín, F. y Rogers, J. D. (1995). Notas sobre la historia, relaciones de hospedante y distribución del género Xylaria (Pyrenomycetes, Sphaeriales) en México. Acta Botánica Mexicana 30: 21-40.

Sir, E. B, Hladki, A. I., Parrado, M. F. y Romero, A. I. (2012). Biodiversity of Xylariaceae (Ascomycota) and their hosts in protected areas from Tucumán (Argentina). Kurtziana 37 (2): 35-48.

Spegazzini, C. L. (1880). Fungi Argentini. Anales Sociedad Científica Argentina 9 (4): 158-192.

Spegazzini, C. L. (1881). Fungi Argentini additis nonnullis brasilensibus montevideensibusque. Anales Sociedad Científica Argentina 12 (3): 97-117.

Spegazzini, C. L. (1889). Fungi Puiggariani. Boletín de la Academia Nacional de Ciencias Córdoba 11 (4):381-622.

Spegazzini, C. L. (1909). Mycetes Argentinenses. Anales del Museo Nacional de Historia Natural de Buenos Aires. Serie 3, 19 (12): 337-350.

Stadler, M., Hawsworth, D. L. y Fournier, J. (2014). The applications of the name Xylaria hypoxylon, based on Clavaria hypoxylonn of Linnaeus. IMA Fungus 5: 57-66.

Thind, K. S. y Waraitch, K. S. (1969). Xylariaceae of India I: Proceedings Academy Indian Sciences 70: 131-138.

Van der Gucht, K. (1995). Ilustrations and descriptions of xylariaceous fungi collected in Papua New Guinea. Bulletin Nationale Plantentuin Belgique 64: 219-403.

Van der Gucht, K. (1996). Xylaria species from Papua New Guinea: cultural and anamorphic studies. Mycotaxon 60: 327-360. 